\title{
\begin{tabular}{l|l} 
Mibraries & DSpace@MIT
\end{tabular}
}

\author{
MIT Open Access Articles
}

Integration of systems biology with organson-chips to humanize therapeutic development

The MIT Faculty has made this article openly available. Please share how this access benefits you. Your story matters.

Citation: Edington, Collin D. et al. “Integration of Systems Biology with Organs-on-Chips to Humanize Therapeutic Development." Edited by Bonnie L. Gray and Holger Becker. Microfluidics, BioMEMS, and Medical Microsystems XV, January - February 2017, San Francisco, California, USA, February 2017 (c) 2017 SPIE

As Published: http://dx.doi.org/10.1117/12.2256078

Publisher: SPIE

Persistent URL: http://hdl.handle.net/1721.1/115077

Version: Final published version: final published article, as it appeared in a journal, conference proceedings, or other formally published context

Terms of Use: Article is made available in accordance with the publisher's policy and may be subject to US copyright law. Please refer to the publisher's site for terms of use. 


\section{Integration of systems biology with organs-on-chips to humanize therapeutic development}

Collin D. Edington, Murat Cirit, Wen Li Kelly Chen, Amanda M. Clark, Alan Wells, et al.

Collin D. Edington, Murat Cirit, Wen Li Kelly Chen, Amanda M. Clark, Alan Wells, David L. Trumper, Linda G. Griffith, "Integration of systems biology with organs-on-chips to humanize therapeutic development," Proc. SPIE 10061, Microfluidics, BioMEMS, and Medical Microsystems XV, 1006113 (28 February 2017); doi: 10.1117/12.2256078

SPIE. Event: SPIE BiOS, 2017, San Francisco, California, United States 


\title{
Integration of systems biology with organs-on-chips to humanize therapeutic development
}

Collin D. Edington ${ }^{\mathrm{a}}$, Murat Cirit ${ }^{\mathrm{a}}$, Wen Li Kelly Chen ${ }^{\mathrm{a}}$, Amanda M. Clark ${ }^{\mathrm{b}}$, Alan Wells ${ }^{\mathrm{b}}$, David L. Trumper $^{\mathrm{a}}$, Linda G. Griffith ${ }^{* a}$

${ }^{a}$ Massachusetts Institute of Technology, 77 Massachusetts Ave. Cambridge MA, USA 02139;

${ }^{\mathrm{b}}$ University of Pittsburgh, 4200 Fifth Ave., Pittsburgh, PA, USA 15260

\begin{abstract}
"Mice are not little people" - a refrain becoming louder as the gaps between animal models and human disease become more apparent. At the same time, three emerging approaches are headed toward integration: powerful systems biology analysis of cell-cell and intracellular signaling networks in patient-derived samples; 3D tissue engineered models of human organ systems, often made from stem cells; and micro-fluidic and meso-fluidic devices that enable living systems to be sustained, perturbed and analyzed for weeks in culture. Integration of these rapidly moving fields has the potential to revolutionize development of therapeutics for complex, chronic diseases, including those that have weak genetic bases and substantial contributions from gene-environment interactions. Technical challenges in modeling complex diseases with "organs on chips" approaches include the need for relatively large tissue masses and organ-organ cross talk to capture systemic effects, such that current microfluidic formats often fail to capture the required scale and complexity for interconnected systems. These constraints drive development of new strategies for designing in vitro models, including perfusing organ models, as well as "mesofluidic" pumping and circulation in platforms connecting several organ systems, to achieve the appropriate physiological relevance.
\end{abstract}

Keywords: organs-on-chips, 3D liver culture, perfusion, drug development, inflammation, organ crosstalk, tissue chip, intestine

\section{INTRODUCTION}

\subsection{Complex diseases require new approaches}

Drug development for cancer, and particularly for chronic inflammatory diseases (e.g. diabetes, arthritis, endometriosis, and Alzheimer's), which typically have weak genetic linkages and poorly-understood, systemic mechanisms where damage accrues over many years, is notoriously difficult. While drug safety failures are typically highlighted in the organs-on-chips literature ${ }^{1,2}$, lack of efficacy is currently the major cause of drug failure overall, and failure rates are higher for complex diseases that have no clear single genetic basis ${ }^{3}$. Such diseases are highly prevalent. For example, the most common cause of abnormal liver function tests in western countries is non-alcoholic fatty liver disease (NAFLD), which has a prevalence of $20-50 \%{ }^{4,5}$; is often co-morbid with Type 2 diabetes; affects tens of millions of individuals worldwide; and is now the second leading cause for liver transplants in the US ${ }^{4,5}$. With many different possible targets in the disease mechanism, and clinical heterogeneity in patient disease characteristics, over a

Microfluidics, BioMEMS, and Medical Microsystems XV, edited by Bonnie L. Gray, Holger Becker, Proc. of SPIE Vol. 10061, 1006113 · C 2017 SPIE · CCC code: 1605-7422/17/\$18 · doi: 10.1117/12.2256078 
dozen new drugs are in the pipeline or clinical trials, joining several established accepted and off-label treatments that have yet to "cure" the symptoms most patients experience ${ }^{6-8}$. Further, at least $10 \%$ of reproductive-age women worldwide experience debilitating pain and infertility due to non-malignant growth of endometrium outside the uterus (endometriosis) or displaced into the uterine muscle (adenomyosis) ${ }^{9,}{ }^{10}$. Current FDA-approved medical therapies attempt to put brakes on estrogen, but a substantial fraction of patients either fail to respond to drugs or suffer severe side effects; surgical removal of lesions helps only a fraction of patients ${ }^{9-11}$. Optimism that new classes of anti-inflammatory drugs developed for other chronic inflammatory diseases such as arthritis has been tempered by the unsuccessful outcomes of recent clinical trials ${ }^{12}$, despite the positive outcomes in pre-clinical animal models of disease ${ }^{13-15}$.

These highly prevalent diseases are examples of how highly heterogeneous patient population and poorlyrepresentative animal models work together to stymie both development and clinical translation of effective new therapies ${ }^{4,5,16}$. One part of the problem in developing effective drugs for such diseases - defining common mechanistic themes among subgroups of patients - is being tackled by innovative integration of multiple -omics measurements across the scales of information flow in cells, from DNA to RNA to protein, protein activity states, and metabolites ${ }^{17}$, as well as similar types of analysis of patient-derived immune cell function ${ }^{18}$. Such approaches led to the first molecular classification of endometriosis patients and insight into the lack of efficacy in JNK inhibitor trials ${ }^{11,19}$ and are yielding insights into type 2 diabetes ${ }^{17,18}$.

Another part of the problem - relative inability to carry out mechanistic studies of new therapies in patients, and lack of animal or in vitro models - is still a major bottleneck. This gap underscores the need for bold new approaches to model these diseases in vitro. Unfortunately, cell culture models, including those derived from human pluripotent stem cells (PSCs), are inadequate: not only do they fail to capture crucial heterogeneous cell interactions within a single organ, development of chronic inflammatory diseases is often the convolution of genetics with lifestyle and environmental exposures, including infection, that are integrated into epigenetic modification of cells throughout the

body ${ }^{17,20}$. Clearly, one step is to develop more complex individual organs-on-chips that capture the local features of disease, especially inflammation. But for systemic diseases affecting multiple organs, organ-on-chips platforms designed to capture physiological interaction phenomena between critical organs systems may yield improvements in understanding both efficacy and off-target effects. Here, we describe examples of how organs-on-chips are being deployed to model complex disease states, and highlight technical challenges in merging disparate fields to design, implement and interpret experiments.

\subsection{Dormancy and growth of metastatic cancer: a challenge beyond the scope of traditional microfluidics?}

The overwhelming majority of breast cancer patients present with no evidence of distant metastatic disease; yet, following removal of the primary tumor and prophylactic chemotherapy to kill disseminated tumor cells throughout the body, a significant fraction will develop tumors years later in the bones, liver, lungs, and brain and succumb to metastatic disease ${ }^{21}$. Triple negative breast cancer (i.e., lacking expression of estrogen, progesterone, and Her2 receptors) is 
particularly deadly: about $25 \%$ of patients die from metastatic disease within 5 years of diagnosis despite aggressive prophylactic chemotherapy ${ }^{22}$. What makes disseminated tumor cells resistant to chemotherapy? What makes these dormant tiny metastatic clusters wake up and grow? These questions cannot be studied in patients - the metastases are not visible -- and animal models fail to capture the key features of human responses, including the increasinglyappreciated role of the human immune system in progression ${ }^{22,23}$.

Numerous 3D models of tumor cells in isolation have been developed in the past few decades ${ }^{24}$, with a notable recent emphasis on how the mechanical microenvironment and geometry of multicellular tumor aggregates influence malignant properties ${ }^{25}$. Similarly, models have emerged to capture tumor-stroma interactions ${ }^{26}$. Microfluidic systems have been deployed to control oxygen and growth factor microenvironments ${ }^{27,} 28$, tumor-endothelial interactions ${ }^{29-31}$ and events in passage of tumor cells from micro-vessels into surrounding tissue ${ }^{32,33}$. While each of these systems captures subsets of tumor behaviors, a significant gap exists in addressing how metastatic nodules of up to several hundred microns interact with human host tissue.

Can microfluidic devices, which typically support culture of tens of thousands of cells, capture the complexity of metastatic resistance to chemotherapy drugs, and the signals that wake up dormant tumors? A single adult human liver lobule comprises about $10^{7}$ cells ${ }^{34}$ and initial metastases are estimated to range from single cells up to a few tens of cells ${ }^{35}$. Thus the ratio of host to metastatic cells in a traditional microfluidic device is substantially skewed, and are more representative of primary tumors or end stage metastases ${ }^{29,36}$. This scaling mismatch may skew tumor cell behaviors, which in vivo are regulated by the overwhelming host signaling milieu and innate inflammatory response ${ }^{37-39}$.

Disease modeling problems such as micrometases that require "meso-scale" tissues, comprising hundreds of thousands to many millions of cells, present interesting challenges in terms of integrating tissue engineering with appropriate pumping and fluidics to provide adequate perfusion rates to such structures, particularly in a user-friendly platform format. Based on the oxygen consumption rate of liver, for example, flow rates of 6-10 $\mu \mathrm{L}$ per million cells per second are needed just to deliver oxygen if cell culture medium is the circulating fluid ${ }^{40-42}$. Another often underappreciated facet of disease modeling with implications for device design is that inflammation - which is a crucial part of a vast number of diseases lacking adequate therapies - is strongly regulated by steroid hormones ${ }^{43}$, 4 , which partition strongly into elastomers such as polydimethylsiloxane (PDMS), making their concentrations difficult to $\operatorname{control}^{45}$.

In order to address these constraints, we developed a 3D micro-perfused liver model aimed at supporting longterm culture of 3D liver-like tissue at scales over a million cells, in a user-friendly format. The heart of the system is a thin $(\sim 0.25 \mathrm{~mm})$ scaffold perforated with an array of $\sim 0.3 \mathrm{~mm}$ diameter channels situated on a membrane support and maintained in a re-circulating flow multi-well plate bioreactor ${ }^{40-42,46}$. Liver cells seeded into the scaffold form 3D tissuelike structures, which are perfused at flow rates sufficient to create a physiological oxygen tension drop across the scaffold without excessive shear ${ }^{40,47}$ and which can be maintained in a functional state for weeks in serum-free culture medium. The reactor system is micro-machined from polysulfone and recirculation is driven by on-board microfluidic pneumatic pumps that are programmed with a user-friendly interface, innovations that are crucial for carrying out 
quantitative pharmaco-kinetic (PK) studies, ease of use in diverse laboratory settings, and adaptation to multi-organ formats. The performance of this system in the context of other liver reactors has been recently reviewed ${ }^{42,48,49}$.

Although we and others have extensively characterized the PK of common small molecule drugs by primary human liver cells in this reactor system ${ }^{50-52}$, standard culture systems are reasonably effective for most small molecule drug PK assays ${ }^{42}$, thus, the kinds of pre-clinical assays driving potential use of this system in the later stages of drug development generally involve the immune system. For example, we recapitulated a complex immunologically-based drug-drug interaction between the anti-IL6 receptor antibody tocilizumab and the metabolism of simvastatin - a phenomenon that could not be reproduced in standard cultures ${ }^{46}$. Inflammation drove dose-dependent suppression of CYP450 activity and metabolism of simvastatin, along with increased production of C-reactive protein (CRP) and cellproduced cytokines and cytokine receptors ${ }^{46}$. Importantly, these responses depend on using physiological levels of cortisol, a steroid hormone, rather than the excessively high concentrations used to boost CYP450 activity in typical hepatocyte cultures ${ }^{43,44}$. This multi-well plate reactor system has also been applied to model NAFLD, including fat accumulation, increased production of adipokines, and suppression of CYP450 activity - which were all modulated by treatment with known drugs ${ }^{53}$.

A potential advantage of the scale of this $3 \mathrm{D}$ perfused liver microreactor system is to first establish micrometastases in the context of a relatively large ( $\geq 1$ million cells) mass of liver cells, and then to analyze complex cell-cell communication network signatures using both measurements that can be routinely made in patients (on the circulating medium) as well as measurements that cannot also be made on patients - the kinetics of tumor cell growth and death. An outstanding challenge in targeted chemotherapeutics is the emergence of resistance to chemotherapy, particularly to inhibitors of specific kinase or growth factor pathways - the tumor initially responds, but surges back after time ${ }^{23}$. Mechanistic systems biology analysis of how intracellular and extracellular signaling networks are connected, probed using in vitro models, have recently been linked to patient outcomes via measurements of proteins shed by tumor cells that appear in the plasma of patients ${ }^{23}$. These models allow some prediction of how patients will respond (or not) to therapies based on a compendium of measurements before and soon after treatment with the drug ${ }^{23}$.

As a first important step to model triple negative breast cancer (TNBC) micrometastases in liver, we established the 3D liver model using primary human hepatocytes and non-parenchymal cells, and seeded the cultures with 100-500 fluorescently-labelled MDA-MB-231 cells as a model TNBC cell line ${ }^{36,54-56}$. Micrometases were established even with the lower limit of cell seeding. Although these cells are typically highly proliferative in standard culture, MDA-MB-231 cells were quiescent in the context of the $3 \mathrm{D}$ liver tissue in the microreactor format - in other words, they exhibited hallmarks of dormancy ${ }^{36,54-56}$. A comprehensive analysis of cytokine and growth factor networks using this model showed that a few tumor cells re-wire the entire culture, and that shifting to a hydrogel scaffold for supporting liver cells provides a more quiescent background liver state than the standard scaffold ${ }^{54-56}$. Interestingly, under standard medium conditions, tumor cells in contact with stiff polystyrene scaffolds are proliferative and responsive to the standard chemotherapy drug doxorubicin, while tumor cells in the $3 \mathrm{D}$ tissue are quiescent and relatively resistant ${ }^{36,54-56}$. 
One potential mechanism for waking dormant tumor cells is inflammation in the liver, which may arise due to increased permeability of the gut from infection, drugs, alcohol, or other causes ${ }^{44}$. As tumor cells and the microenvironment can respond to inflammatory cues ${ }^{36}$, leaky gut may lead to stimulation of dormant micrometastases. To model this, we adapted the Liverchip platform to link a transwell gut module with the liver module, using the same on-board pumping technology to drive fluidic communication between the two modules as well as circulation within each module. A gut MPS with features of innate immunity was created by seeding a 9:1 mix of absorptive enterocytes (CC2BB/e1 line) and mucin-secreting goblet cells (HT29-MTX line) (adapted from ${ }^{57}$ ) on the apical surface and dendritic cells, obtained from in vitro differentiation of human PBMCs-derived monocytes, on the lower side of the membrane. After 21 days, the epithelial layer exhibited trans-epithelial electrical resistance (TEER) values $>250$ $\Omega \bullet \mathrm{cm}^{2}$, produced mucus (as assessed by Alcian blue ${ }^{58}$ ), and was penetrated by extensions of dendritic cells. These interconnected organ systems show synergistic responses to inflammation ${ }^{59}$ and establish a model for future studies of gut-derived inflammation on cancer micrometases in liver.

\subsection{Frontiers in multi-organ systems: integrating minimal physiological models with hardware}

Multi-organ platforms are just beginning to emerge as potential tools for disease modeling and drug development, and many challenges remain: first, in defining where the information from such systems justifies the added cost, as each organ system comprises a mix of expensive primary human cells, and second, in defining the appropriate "minimal set" of interacting organ systems to represent a disease state. Existing multi-organ platforms that might be used for disease models often fail to meet requirements for quantitative PK due to use of polydimethylsiloxane (PDMS) and highly constrained pumping or fluid transport schemes ${ }^{60,61}$, large circulation volumes that preclude detection of metabolites and cell-produced proteins ${ }^{62,63}$, or are geared more toward pre-clinical tests of metabolism and toxicity and thus lack organ complexity ${ }^{1,2}$. Hence, there is tremendous room for innovation in integration of the hardware, biology, and modeling.

A crucial link in this regard is the emerging field of Quantitative Systems Pharmacology (QSP). QSP combines experimental and computational approaches to pharmacological concepts ${ }^{64,65}$ and is being extended to design and interpretation of MPS technologies ${ }^{50,66-68}$. While a variety of pharmacokinetic and pharmacodynamic (PKPD) models are used to define relationships between drug kinetics and biological effects in vivo, PKPD models for MPS analysis must capture the interrelated physical dynamics (e.g., flow rates in the MPS) and biological dynamics (e.g., cytokine/growth factor/hormone production and release) in order to select the appropriate experimental conditions, analyze results, and predict human outcomes. I.e., they must use physiologically-based PK (PBPK) models. In vitro to in vivo translation (IVIVT) is an interpretive step that compares and validates MPS results to clinically-relevant outcomes. While in vivo to in vitro correlation (IVIVC) and in vivo to in vitro extrapolation (IVIVE) methods have been widely used to predict PK, IVIVT goes a step further to include analysis of endogenous growth factor, inflammatory and hormone signals that affect function. Thus, IVIVT approaches can additionally predict PD, clinical toxicology, biomarkers, and patient stratification using information from MPS technologies. PBPK models for IVIVT can 
quantitatively forecast human responses, accounting for missing organs, organ and media size mismatches, and drug exposure.

\section{CONCLUSION}

\subsection{Application-based approaches}

There are no 'one size fits all' solutions for modeling complex human disease "on a chip", and each model will need to be tailored to its intended application, capturing the minimum biological complexity in order produce translatable biological outputs. Tackling the challenges of integrating multi-organ systems in a way that yields meaningful advances will therefore require bringing together groups with diverse expertise in biology, engineering, and systems pharmacology.

\section{ACKNOWLEDGEMENTS}

This work was supported by the DARPA Microphysiological Systems Program (W911NF-12-2- 0039) and NIH UH3 TR000496.

\section{REFERENCES}

[1] Sung, J. H., Esch, M. B., Prot, J.-M., Long, C. J., et al., "Microfabricated mammalian organ systems and their integration into models of whole animals and humans," Lab on a Chip 13, 1201-1212 (2013).

[2] Caplin, J. D., Granados, N. G., James, M. R., Montazami, R., et al., "Microfluidic Organ-on-a-Chip Technology for Advancement of Drug Development and Toxicology," Advanced Healthcare Materials 4, 1426-1450 (2015).

[3] Cook, D., Brown, D., Alexander, R., March, R., et al., "Lessons learned from the fate of AstraZeneca's drug pipeline: a five-dimensional framework," Nat Rev Drug Discov 13, 419-31 (2014).

[4] Anstee, Q., Targher, G. and Day, C., "Progression of NAFLD to diabetes mellitus, cardiovascular disease, or cirrhosis," Nat Rev Gastroenterol Hepatol 10, 330-344 (2013).

[5] Brunt, E. M., Wong, V. W., Nobili, V., Day, C. P., et al., "Nonalcoholic fatty liver disease," Nat Rev Dis Primers 1, 15080 (2015).

[6] Cassidy, S. and Syed, B. A., "Nonalcoholic steatohepatitis (NASH) drugs market," Nat Rev Drug Discov 15, 745-746 (2016).

[7] DeFronzo, R., Tripathy, D., Schwenke, D., Banerji, M., et al., "Prevention of Diabetes With Pioglitazone in ACT NOW Physiologic Correlates," Diabetes 62, 3920-3926 (2013).

[8] Stafford, J. and Elasy, T., "Treatment update: thiazolidinediones in combination with metformin for the treatment of type 2 diabetes," VascHealth Risk Management 3, 503-510 (2007).

[9] Vercellini, P., Viganò, P., Somigliana, E. and Fedele, L., "Endometriosis: pathogenesis and treatment," Nature Publishing Group 10, 261-275 (2013).

[10] Benagiano, G., Habiba, M., Brosens, I., Habiba, M., et al., "The pathophysiology of uterine adenomyosis: An update," Fertility and Sterility 98, 572-579 (2012).

[11] Beste, M. T., Pfäffle-Doyle, N., Prentice, E. A., Morris, S. N., et al., "Molecular network analysis of endometriosis reveals a role for c-Jun-regulated macrophage activation.," Science Translational Medicine 6, 222ra16 (2014).

[12] Koninckx, P. R., Craessaerts, M., Timmerman, D., Cornillie, F., et al., "Anti-TNF-alpha treatment for deep endometriosis-associated pain: a randomized placebo-controlled trial," Hum Reprod 23, 2017-23 (2008).

[13] Hussein, M., Chai, D. C., Kyama, C. M., Mwenda, J. M., et al., "c-Jun NH2-terminal kinase inhibitor bentamapimod reduces induced endometriosis in baboons: an assessor-blind placebo-controlled randomized study," Fertil Steril $105,815-24$ e5 (2016). 
[14] D'Hooghe, T. M., Nugent, N. P., Cuneo, S., Chai, D. C., et al., "Recombinant human TNFRSF1A (r-hTBP1) inhibits the development of endometriosis in baboons: a prospective, randomized, placebo- and drug-controlled study," Biol Reprod 74, 131-6 (2006).

[15] Grund, E. M., Kagan, D., Tran, C. A., Zeitvogel, A., et al., "Tumor necrosis factor-alpha regulates inflammatory and mesenchymal responses via mitogen-activated protein kinase kinase, $\mathrm{p} 38$, and nuclear factor kappaB in human endometriotic epithelial cells," Mol Pharmacol 73, 1394-404 (2008).

[16] Maher, J. J., "Modeling fatty liver disease in animals: Is there an optimal approach, and is the effort worthwhile?," Hepatology 64, 1398-1400 (2016).

[17] Lee, S., Zhang, C., Kilicarslan, M., Piening, B. D., et al., "Integrated Network Analysis Reveals an Association between Plasma Mannose Levels and Insulin Resistance," Cell Metab 24, 172-84 (2016).

[18] Ip, B., Cilfone, N. A., Belkina, A. C., DeFuria, J., et al., "Th17 cytokines differentiate obesity from obesityassociated type 2 diabetes and promote TNFalpha production," Obesity (Silver Spring) 24, 102-12 (2016).

[19] Miller, M. A., Meyer, A. S., Beste, M. T., Lasisi, Z., et al., "ADAM-10 and -17 regulate endometriotic cell migration via concerted ligand and receptor shedding feedback on kinase signaling.," Proceedings of the National Academy of Sciences 110, E2074-E2083 (2013).

[20] Bruner-Tran, K., Ding, T. and Osteen, K., "Dioxin and Endometrial Progesterone Resistance," Semin Reprod Med 28, 059-068 (2010).

[21] Lu, X. and Kang, Y., "Organotropism of breast cancer metastasis," J Mammary Gland Biol Neoplasia 12, 153-62 (2007).

[22] Kalimutho, M., Parsons, K., Mittal, D., Lopez, J. A., et al., "Targeted Therapies for Triple-Negative Breast Cancer: Combating a Stubborn Disease," Trends Pharmacol Sci 36, 822-46 (2015).

[23] Miller, M. A., Oudin, M. J., Sullivan, R. J., Wang, S. J., et al., "Reduced Proteolytic Shedding of Receptor Tyrosine Kinases Is a Post-Translational Mechanism of Kinase Inhibitor Resistance," Cancer Discov 6, 382-99 (2016).

[24] Unger, C., Kramer, N., Walzl, A., Scherzer, M., et al., "Modeling human carcinomas: physiologically relevant 3D models to improve anti-cancer drug development," Adv Drug Deliv Rev 79-80, 50-67 (2014).

[25] Gilbert, P. and Weaver, V., "Cellular adaptation to biomechanical stress across length scales in tissue homeostasis and disease," Semin Cell Dev Biol. In Press, (2016).

[26] Rothberg, J. M., Sameni, M., Moin, K. and Sloane, B. F., "Live-cell imaging of tumor proteolysis: impact of cellular and non-cellular microenvironment," Biochim Biophys Acta 1824, 123-32 (2012).

[27] Sung, K. E. and Beebe, D. J., "Microfluidic 3D models of cancer," Adv Drug Deliv Rev 79-80, 68-78 (2014).

[28] Stroock, A. D. and Fischbach, C., "Microfluidic culture models of tumor angiogenesis," Tissue Engineering Part A 16, 2143-2146 (2010).

[29] Sobrino, A., Phan, D. T., Datta, R., Wang, X., et al., "3D microtumors in vitro supported by perfused vascular networks," Sci Rep 6, 31589 (2016).

[30] Wang, X., Phan, D. T., Sobrino, A., George, S. C., et al., "Engineering anastomosis between living capillary networks and endothelial cell-lined microfluidic channels," Lab Chip 16, 282-90 (2016).

[31] Roudsari, L. C. and West, J. L., "Studying the influence of angiogenesis in in vitro cancer model systems," Adv Drug Deliv Rev 97, 250-9 (2016).

[32] Zervantonakis, I. K., Hughes-Alford, S. K., Charest, J. L., Condeelis, J. S., et al., "Three-dimensional microfluidic model for tumor cell intravasation and endothelial barrier function," Proc Natl Acad Sci U S A 109, 13515-20 (2012).

[33] Jeon, J. S., Bersini, S., Gilardi, M., Dubini, G., et al., "Human 3D vascularized organotypic microfluidic assays to study breast cancer cell extravasation," Proc Natl Acad Sci U S A 112, 214-9 (2015).

[34] Bianconi, E., Piovesan, A., Facchin, F., Beraudi, A., et al., "An estimation of the number of cells in the human body," Ann Hum Biol 40, 463-71 (2013).

[35] Au, S. H., Storey, B. D., Moore, J. C., Tang, Q., et al., "Clusters of circulating tumor cells traverse capillary-sized vessels," Proc Natl Acad Sci U S A 113, 4947-52 (2016).

[36] Clark, A. M., Ma, B., Taylor, D. L., Griffith, L., et al., "Liver metastases: Microenvironments and ex-vivo models," Exp Biol Med (Maywood) 241, 1639-52 (2016).

[37] Chao, Y., Wu, Q., Acquafondata, M., Dhir, R., et al., "Partial Mesenchymal to Epithelial Reverting Transition in Breast and Prostate Cancer Metastases," Cancer Microenvironment 5, 19-28 (2011).

[38] Chao, Y., Wu, Q., Shepard, C. and Wells, A., "Hepatocyte induced re-expression of E-cadherin in breast and prostate cancer cells increases chemoresistance," Clin Exp Metastasis 29, 39-50 (2011). 
[39] Chao, Y. L., Shepard, C. R. and Wells, A., "Breast carcinoma cells re-express E-cadherin during mesenchymal to epithelial reverting transition," Mol Cancer 9, 179 (2010).

[40] Powers, M. J., Domansky, K., Kaazempur-Mofrad, M. R., Kalezi, A., et al., "A microfabricated array bioreactor for perfused 3D liver culture," Biotechnol Bioeng 78, 257-69 (2002).

[41] Domansky, K., Inman, W., Serdy, J., Dash, A., et al., "Perfused multiwell plate for 3D liver tissue engineering," Lab on a Chip 10, 51-58 (2010).

[42] Ebrahimkhani, M. R., Nieman Shepard, J. A., Raredon, M. S. B., Hughes, D. J., et al., "Bioreactor Technologies to Support Liver Function In Vitro," Advanced Drug Delivery Reviews Apr, 132-57

(2014).

[43] Sarkar, U., Rivera-Burgos, D., Large, E. M., Hughes, D. J., et al., "Metabolite profiling and pharmacokinetic evaluation of hydrocortisone in a perfused three-dimensional human liver bioreactor," Drug Metab Dispos 43, 1091-9 (2015).

[44] Dash, A., Inman, W., Hoffmaster, K., Sevidal, S., et al., "Liver tissue engineering in the evaluation of drug safety.," Expert Opin Drug Metab Toxicol 5, 1159-1174 (2009).

[45] Regehr, K. J., Domenech, M., Koepsel, J. T., Carver, K. C., et al., "Biological implications of polydimethylsiloxanebased microfluidic cell culture," Lab on a Chip 9, 2132-2139 (2009).

[46] Long, T., Cosgrove, P., Dunn, R. n., Stolz, D., et al., "Modeling Therapeutic Antibody-Small Molecule Drug-Drug Interactions Using a Three Dimensional Perfusable Human Liver Coculture Platform," Drug Metab Dispos 44, 1940-1948 (2016).

[47] Yates, C., Shepard, C. R., Papworth, G., Dash, A., et al., "Novel three-dimensional organotypic liver bioreactor to directly visualize early events in metastatic progression.," Adv. Cancer Res. 97, 225-246 (2007).

[48] Usta, O. B., McCarty, W. J., Bale, S., Hegde, M., et al., "Microengineered cell and tissue systems for drug screening and toxicology applications: Evolution of in-vitro liver technologies," Technology (Singap World Sci) 3, 1-26 (2015).

[49] Griffith, L. G., Wells, A. and Stolz, D. B., "Engineering liver," Hepatology 60, 1426-1434 (2014).

[50] Tsamandouras, N., Kostrzewski, T., Stokes, C., Griffith, L., et al., "Quantitative assessment of population variability in hepatic drug metabolism using a perfused 3D human liver microphysiological system.," J Pharmacol Exp Ther. 360, 95-105 (2016).

[51] Vivares, A., Salle-Lefort, S., Arabeyre-Fabre, C., Ngo, R., et al., "Morphological behaviour and metabolic capacity of cryopreserved human primary hepatocytes cultivated in a perfused multiwell device," Xenobiotica 45, 29-44 (2015).

[52] Sarkar, U., Rivera-Burgos, D., Large, E. M., Hughes, D. J., et al., "Metabolite Profiling and Pharmacokinetic Evaluation of Hydrocortisone in a Perfused Three-Dimensional Human Liver Bioreactor," Drug Metab Dispos 43, 1091-1099 (2015).

[53] Kostrzewski, T., Cornforth, T., Snow, S., Ouro-Gnao, L., et al., "Three-dimensional perfused human in vitro model of non-alcoholic fatty liver disease," World J Gastroenterol Accepted for publication, (2016).

[54] Wheeler, S. E., Clark, A. M., Taylor, D. P., Young, C. L., et al., "Spontaneous dormancy of metastatic breast cancer cells in an all human liver microphysiologic system," Br J Cancer 111, 2342-50 (2014).

[55] Neiman, J. A. S., Raman, R., Chan, V., Rhoads, M. G., et al., "Photopatterning of Hydrogel Scaffolds Coupled to Filter Materials Using Stereolithography for Perfused 3D Culture of Hepatocytes," Biotechnology and Bioengineering 112, 777-787 (2015).

[56] Clark, A., Wheeler, S., Young, C., Stockdale, L., et al., "A liver microphysiological system of tumor cell dormancy and inflammatory responsiveness is affected by scaffold properties," Lab Chip 17, 156-168 (2017).

[57] Mahler, G. J., Esch, M. B., Tako, E., Southard, T. L., et al., "Oral exposure to polystyrene nanoparticles affects iron absorption," Nat Nanotechnol 7, 264-71 (2012).

[58] Hall, R., Miller, R., Peatfield, A. and Richardson, P., "A colorimetric assay for mucous glycoproteins using Alcian Blue," Biochem Soc Trans. 8, 72 (1980).

[59] Chen, W. L. K., Edington, C., Suter, E., Yu, J., et al., "Integrated Gut/Liver Microphysiological Systems Platform Elucidates Inflammatory Cytokine/Chemokine Inter-Tissue Crosstalk," Submitted, (2017).

[60] Wagner, I., Materne, E.-M., Brincker, S., Süßbier, U., et al., "A dynamic multi-organ-chip for long-term cultivation and substance testing proven by 3D human liver and skin tissue co-culture," Lab Chip 13, 3538 (2013).

[61] Maschmeyer, I., Lorenz, A. K., Schimek, K., Hasenberg, T., et al., "A four-organ-chip for interconnected long-term co-culture of human intestine, liver, skin and kidney equivalents," Lab Chip 15, 2688-99 (2015). 
[62] Iori, E., Vinci, B., Murphy, E., Marescotti, M. C., et al., "Glucose and fatty acid metabolism in a 3 tissue in-vitro model challenged with normo- and hyperglycaemia," PLoS One 7, e34704 (2012).

[63] Guzzardi, M. A., Domenici, C. and Ahluwalia, A., "Metabolic control through hepatocyte and adipose tissue crosstalk in a multicompartmental modular bioreactor," Tissue Eng Part A 17, 1635-42 (2011).

[64] Knight-Schrijver, V. R., Chelliah, V., Cucurull-Sanchez, L. and Le Novere, N., "The promises of quantitative systems pharmacology modelling for drug development," Comput Struct Biotechnol J 14, 363-370 (2016).

[65] Yang, R., Niepel, M., Mitchison, T. K. and Sorger, P. K., "Dissecting variability in responses to cancer chemotherapy through systems pharmacology," Clin Pharmacol Ther 88, 34-8 (2010).

[66] Abaci, H. E. and Shuler, M. L., "Human-on-a-chip design strategies and principles for physiologically based pharmacokinetics/pharmacodynamics modeling," Integr Biol (Camb) 7, 383-91 (2015).

[67] Stokes, C. L., Cirit, M. and Lauffenburger, D. A., "Physiome-on-a-Chip: The Challenge of 'Scaling' in Design, Operation, and Translation of Microphysiological Systems," CPT: Pharmacometrics \& Systems Pharmacology 4, 559-562 (2015).

[68] Yu, J., Cilfone, N. A., Large, E. M., Sarkar, U., et al., "Quantitative Systems Pharmacology Approaches Applied to Microphysiological Systems (MPS): Data Interpretation and Multi-MPS Integration," CPT Pharmacometrics Syst. Pharmacol. 4, 585-94 (2015). 